\section{Gute Klinische Praxis}

U. Zimmermann ${ }^{1}$ und A. Steinhorst ${ }^{2}$

${ }^{1}$ Gesundheit / Forensik, Deutsche Akkreditierungsstelle

GmbH (DAkkS), Frankfurt, Deutschland

${ }^{2}$ EA Secretariat, Paris, Frankreich

\section{$\operatorname{Synonym(e)~GCP~}$}

Englischer Begriff good clinical practice (GCP)

Beschreibung Die Gute Klinische Praxis umfasst einen Katalog international anerkannter ethischer und wissenschaftlicher Qualitätsanforderungen, die bei der Planung, Durchführung und Aufzeichnung klinischer Prüfungen an Menschen sowie der Berichterstattung über diese Prüfungen eingehalten werden müssen. Die Einhaltung dieser Praxis gewährleistet, dass die Rechte, die Sicherheit und das Wohlergehen der Teilnehmer an klinischen Prüfungen geschützt werden und dass die Ergebnisse der klinischen Prüfungen glaubwürdig sind.

Zur Überprüfung der Übereinstimmung mit den Bestimmungen zur guten klinischen Praxis und zur guten Herstellungspraxis benennen die Mitgliedstaaten Inspektoren, die die Aufgabe haben, in den an einer klinischen Prüfung beteiligten Stellen, insbesondere in der Prüfstelle bzw. den Prüfstellen, am Herstellungsort des Prüfpräparats, in allen an der Prüfung beteiligten Laboratorien und/oder in den Einrichtungen des Sponsors, Inspektionen durchzuführen.

\section{Literatur}

Richtlinie 2001/02/EG des europäischen Parlaments und des Rates vom 4. April 2001 zur Angleichung der Rechts- und Verwaltungsvorschriften der Mitgliedstaaten über die Anwendung der guten klinischen Praxis bei der Durchführung von klinischen Prüfungen mit Humanarzneimitteln 